\title{
Caracterización de la superaleación ODS MA 956 para aplicaciones biomédicas
}

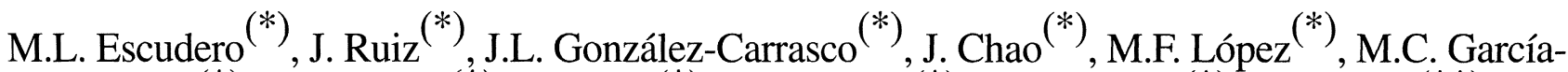 \\ Alonso $^{(*)}$, H. Canahua ${ }^{(*)}$, P. Adeva ${ }^{(*)}$, A.G. Coedo ${ }^{(*)}$, M.T. Dorado ${ }^{(*)}$, J.C. Rubio $^{(* *)}$, \\ M.E. Martínez ${ }^{(* *)}$, L. Munuera ${ }^{(* *)}$, D. de Agustín ${ }^{(* *)}$ y J. Ruiz ${ }^{(* *)}$
}

Resumen Desde que la MA 956 fue propuesta como posible nuevo biomaterial en base a su buena resistencia a la corrosión frente a sueros fisiológicos, un largo camino, aún sin finalizar, se ha recorrido, en el que se ha llevado a cabo una caracterización exhaustiva de esta aleación a temperatura ambiente con fines tecnológicos bien definidos: su posible uso en aplicaciones biomédicas, bien sea como prótesis de cadera o de rodilla y para implantes dentales. Los estudios se han llevado a cabo comparando esta aleación con los materiales más comúnmente utilizados en implantes ortopédicos como aleaciones de titanio y polietileno.

Palabras clave: Biomaterial. Corrosión. Desgaste. Implantes metálicos.

\section{Characterization of the ODS MA 956 superalloy for biomedical surgical implants}

\begin{abstract}
Since the MA 956 was proposed as a possible new biomaterial due to its good corrosion resistance values in physiological fluids a long way, still not finished, has been done. An exhaustive characterization of this alloy at room temperature has been developed. The technological objetives are well established: the possible use of this material for biomedical applications as hip or knee prostheses and as dental implants. The study was performed comparing the results of this alloy with the materials used nowadays as surgical implants, i.e., titanium alloys and polyethylene.
\end{abstract}

Keywords: Biomaterial. Corrosion. Wear. Metallic implants.

\section{INTRODUCCIÓN}

Uno de los grandes retos en el campo de los biomateriales es conseguir materiales que presenten buenas propiedades de resistencia frente a la corrosión, sin renunciar a las propiedades mecánicas. Los materiales para implantes deben soportar los esfuerzos físicos del órgano a reemplazar y además deben resistir el ataque del medio corrosivo, produciendo una baja toxicidad. Para cubrir estas necesidades se recubren los materiales metálicos con diferentes recubrimientos como alúmina, hidroxiapatita, carbono, nitruros, etc.

(*) Centro Nacional de Investigaciones Metalúrgicas, CENIM (CSIC), Avda. de Gregorio del Amo, 8. 28040Madrid (España).

(**) Hospital La Paz. Po. de la Castellana, 261. 28046-Madrid (España).

(***) Hospital Militar Central Gómez Ulla. Glorieta del Ejército, s/n. 28047-Madrid (España).

\section{PARTE EXPERIMENTAL}

La MA 956 es una superaleación ferrítica procesada vía pulvimetalúrgica por aleado mecánico (MA) y mediante tratamientos termomecánicos. Su composición química es $\mathrm{Fe}-20 \mathrm{Cr}-5 \mathrm{Al}-0,5 \mathrm{Y}_{2} \mathrm{O}_{3}(\%$ en masa). Los tratamientos de oxidación térmica fueron llevados a cabo a $1.100^{\circ} \mathrm{C}$ durante $100 \mathrm{~h}$ en aire. En estas condiciones, se forma una capa superficial de $\alpha-\mathrm{Al}_{2} \mathrm{O}_{3}$ de aproximadamente $5 \mu \mathrm{m}$ de espesor. La composición química, expresada en \% en masa, de la aleación de base titanio es Ti-6Al-4V.

Los estudios de resistencia a la corrosión se realizaron mediante técnicas electroquímicas sumergiendo las probetas en un electrólito similar al fluido fisiológico.

Los ensayos de tracción se llevaron a cabo en un rango de velocidades de $10^{-5}$ a $10^{-1} \mathrm{~s}^{-1}$ sobre muestras de $3 \mathrm{~mm}$ de diámetro y $10 \mathrm{~mm}$ de longitud. 
Los ensayos de desgaste se realizaron en seco con una presión de contacto de $80 \mathrm{MPa}$ y a una velocidad de $24 \mathrm{rpm}$. Las muestras eran cilíndricas de $10 \mathrm{~mm}$ de ancho y de $35 \mathrm{~mm}$ de diámetro.

Los ensayos in vivo se llevaron a cabo sobre ratas Wistar de 3 meses, a las que se les insertaban probetas de $1 \mathrm{~mm}$ de diámetro y $5 \mathrm{~mm}$ de longitud en la zona esponjosa de la parte distal del fémur.

\section{RESULTADOS Y DISCUSIÓN}

Desde el punto de vista de la corrosión, la aleación MA956, preoxidada in situ, presenta una resistencia a la corrosión en los fluidos orgánicos tan elevada que es difícil de determinar cuantitativamente. Se puede hablar de umbrales de resistencia de transferencia, $R_{\mathrm{t}}$, por encima de $10^{8} \Omega(1$ y 2$)$. La resistencia a la picadura es también completamente satisfactoria. En la figura 1 se observa que las densidades de corriente están en el rango de $10^{-9}$ $\mathrm{A} \mathrm{cm}{ }^{-2}$, lo que se encuentra, en muchos casos, en el límite de la sensibilidad de medida de los aparatos, 1 nA.

Con respecto al titanio, el umbral de pasivación es de $10^{-6} \mathrm{~A} \mathrm{~cm}^{-2}$, unos tres órdenes de magnitud superior al de la MA 956 oxidada (3). Como se observa en la figura 1 , la probabilidad de rotura de la capa es pequeña, pero, en caso de que dicha rotura se produjera, la repasivación está asegurada debido al elevado contenido de cromo de la aleación.

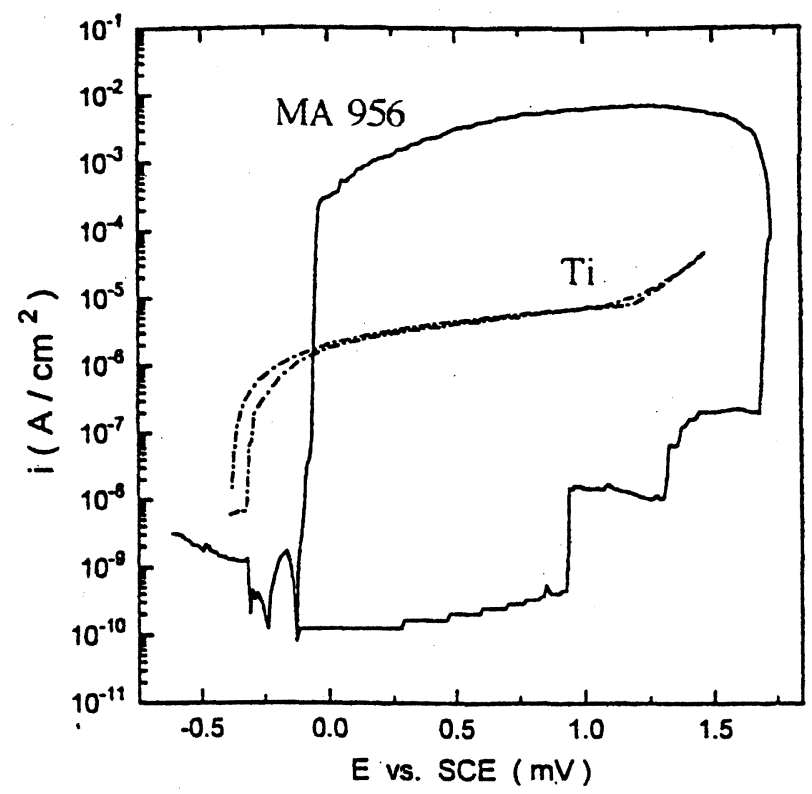

FIG. 1.-Curvas de polarización anódica para la aleación MA 956 preoxidada y para la aleación de base titanio.

FIG. 1.- Anodic polarization curves for the preoxidized MA 956 and titanium alloys.
Dicha confirmación se llevó a cabo por medidas de espectrometría de emisión fotoelectrónica XPS (4).

Para evaluar la excelente adherencia de la capa al sustrato metálico, se midieron indirectamente las tensiones residuales en la capa, que alcanzan un valor estimado del orden de los 5.000 MPa. Dichos ensayos se llevaron a cabo mediante el cálculo del límite elástico en función de la velocidad de deformación para probetas con y sin capa de alúmina, como queda reflejado en la figura 2 (5).

El comportamiento al desgaste de esta aleación queda resumido en la figura 3. El mejor comportamiento es el ofrecido por el par MA 965 oxidadapolietileno con coeficientes de fricción muy bajos después de $5 \times 10^{6}$ ciclos. Estos buenos resultados implicarían un aumento en el tiempo de servicio de aquellas zonas de prótesis articulares sometidas a fricción, donde la cabeza de la articulación podría ser de la aleación MA 956 oxidada y la cúpula de polietileno (6).

Un ejemplo de la respuesta in vivo de esta aleación preoxidada se muestra en la micrografía del SEM de la figura 4, en la que se observa que la integración de la prótesis al hueso ha sido excelente, con una neoformación de hueso abrazando al implante completamente satisfactoria (7).

\section{CONCLUSIONES}

- La MA 956 preoxidada in situ con capas de alúmina en torno a las $5 \mu \mathrm{m}$ de espesor ofrece una resistencia a la corrosión en general y a la susceptibilidad a la corrosión por picaduras muy superior a la de cualquier biomaterial metálico convencional.

- Las elevadas tensiones residuales de compresión previenen la formación de grietas durante la

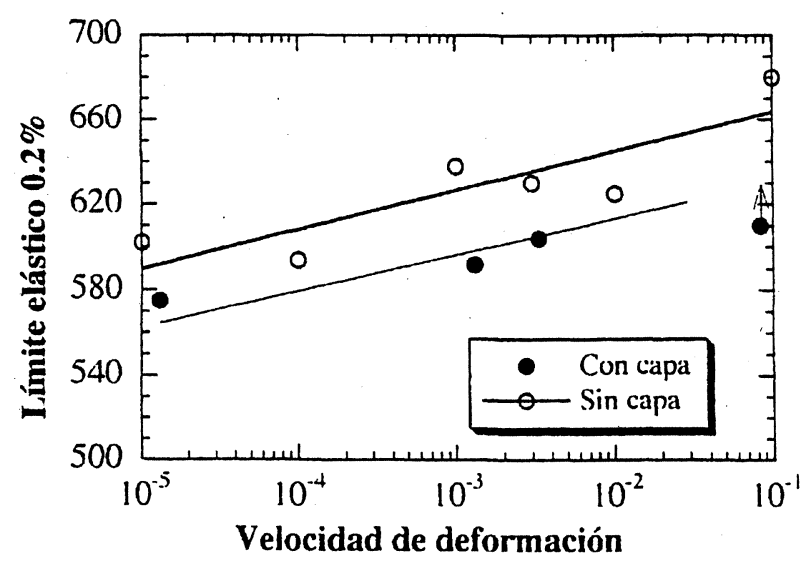

FIG. 2.- Límite elástico para muestras con capa y sin ella frente a la velocidad de deformación.

FIG. 2.- Yield stress for samples with scale and scale-free vs strain rate. 


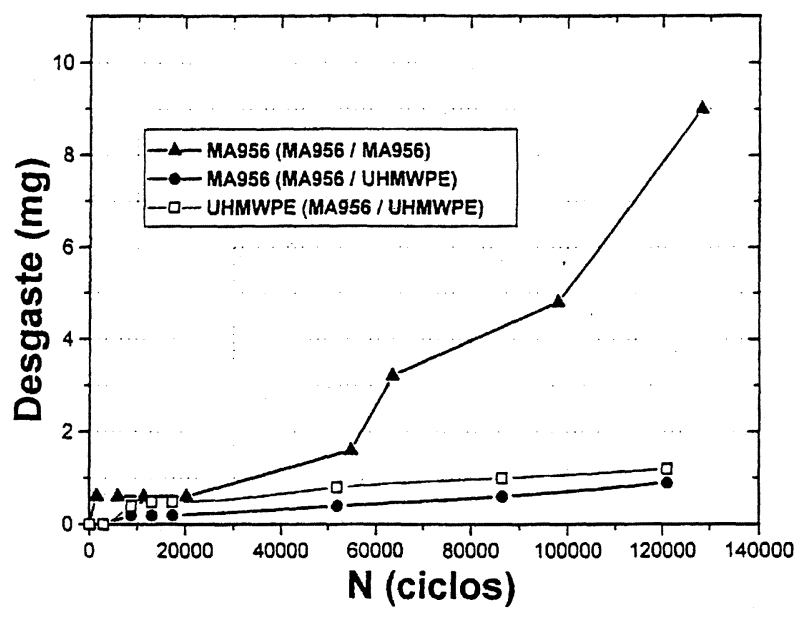

FIG. 3.- Comportamiento al desgaste en función del número de ciclos.

FIG. 3.- Wear behaviour as a function of the number of cycles.

deformación elástica, lo que favorece una buena resistencia al desgaste.

- De los pares ensayados, en lo que a resistencia al desgaste se refiere, el mejor es el formado por la aleación MA 956 preoxidada y el polietileno.

- La capa de alúmina induce una respuesta in vivo completamente satisfactoria, con un grado de biocompatibilidad excelente.

\section{Agradecimiento}

Este trabajo ha sido realizado gracias a la financiación, por parte de la CICYT, del proyecto MAT0249, que consta de tres subproyectos 01, 02 y 03.

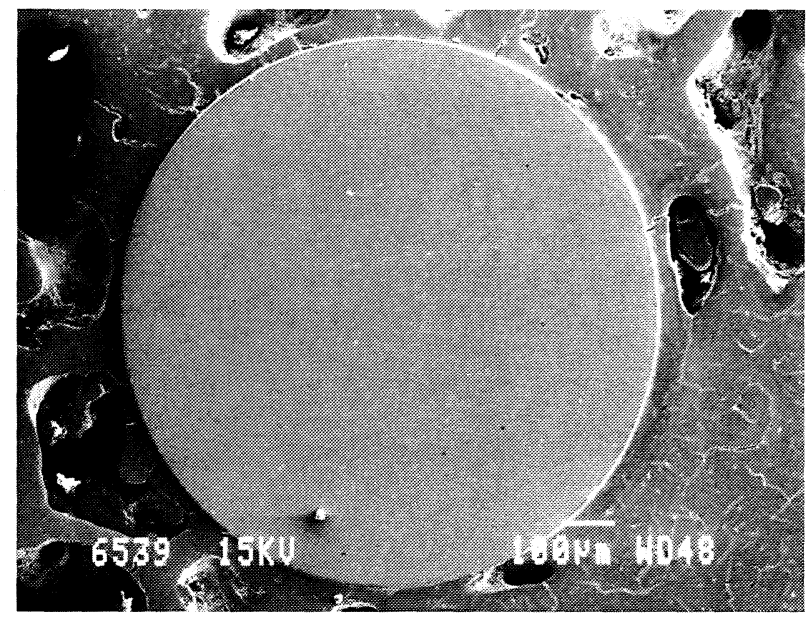

FIG. 4.- Micrografía de SEM de la parte distal del fémur con aleación MA 956 preoxidada, a los tres meses de su implantación.

FIG. 4.-SEM micrograph of distal end of the femur with preoxidized MA 956 alloy after 3 months of implantation.

\section{REFERENCIAS}

(1) Escudero, M.L. y GonZalez-Carrasco, J.L. Biomaterials, 15, 1994: 1.175-1.180.

(2) Escudero, M.L., Gonzalez-Carrasco, J.L., GarciaAlonso, C. y Ramirez, E. Biomaterials, 16, 1995: 735740.

(3) Escudero, M.L., Lopez, M.F., Ruiz, J., Garcia-Alonso, M.C. y Canahua, H. J. Biomedical Mater. Res., 31, 1996:313-317.

(4) LóPez, M.F., Gutiérrez, A., Garcia-Alonso, M.C. y Escudero, M.L. J. Mater. Res., 1998 (Pendiente de publicación).

(5) Chao, J., Gonzalez-Carrasco, J.L., Ibañez, J., Escudero, M.L. y Gonzalez-Doncel, G. Metall. Mater. Trans., 27A, 1996: 3.809-3.816. 